\title{
Evaluación del Fondo Social Europeo 2007-2013 en el contexto de la política de cohesión europea y la crisis
}

\section{Evaluation of the European Social Fund 2007-2013 in the context of European cohesion policy and the global crisis}

Núm. 7 (2016), pp. 48-63

Pérez Viejo, Jesús ${ }^{* 1}$

Castro Clemente, Concepción ${ }^{* 2}$

Recibido: abril, 2016

Aceptado: septiembre, 2016

JEL Clasif: I3, I31

DOI: $10.5944 /$ reppp.7.2016.16304

\footnotetext{
*1 Jesús Pérez Viejo. Licenciado en Psicología, Doctor en Trabajo Social, Máster Oficial en Trabajo Social, en Sexualidad, experto en Género, Derechos Humanos y Violencia. Profesor asociado Universidad Carlos III de Madrid, docente de diferentes Máster universitarios y con amplia experiencia en la formación de profesionales de entidades públicas y privadas en temas relacionados con la violencia, el counsseling, las habilidades de relación de ayuda y los autocuidados en equipos profesionales, a nivel nacional e internacional (Chihuahua, México, Bruselas, Francia, Italia y España). Más de 15 años de experiencia en el sector de las ONG, desarrollando fundamentalmente funciones de coordinación y dirección. E-mail: jeperezv@clio.uc3m.es

*2 Concepción Castro Clemente, Graduada y Diplomada en Trabajo Social. Máster Oficial Universidad Pontificia Comillas «Investigación sobre familias. Perspectivas psicológicas, educativas y sociales». Profesora-Tutora de Grado Trabajo Social en Madrid y colaboradora en diferentes Másteres en Universidad Nacional de Educación a Distancia (UNED). Amplia experiencia en el trabajo con grupos y en el desarrollo de proyectos nacionales y europeos con jóvenes en el contexto de la formación no formal. Coautor y colaborador de varios libros, capítulos y artículos de investigación relacionados con la especialidad. Miembro participante en varias asociaciones de Trabajo Social de ámbito nacional e internacional. Actualmente Doctoranda en la Universidad Pontificia de Comillas. E-mail: concastro@madrid.uned.es
} 


\title{
Resumen
}

La política de cohesión social se define como un marco político supranacional de solidaridad social y cooperación competitiva que se traduce en cientos de miles proyectos financiados por el Fondo Europeo de Desarrollo Regional (FEDER) y el Fondo Social Europeo. Una finalidad principal de estos instrumentos financieros es la construcción de capital físico y capital humano para que regiones y poblaciones europeas afectadas por la exclusión económica y social, puedan construir y desplegar las capacidades y competencias necesarias dirigidas al logro de un nivel socio económico digno y coherente a través de sus incorporaciones como actores activos dentro del sistema productivo y en el mercado de trabajo. Lo que diferencia a la política de cohesión social europea de otras políticas, es que su configuración jurídica y financiera no subsidia asistencialmente la pobreza, su propósito es actuar sobre las causas estructurales de la exclusión socioeconómica. La cohesión económica y social descrita por los Estados miembros en el Acta Única Europea de 1986 se propone reducir las brechas estructurales entre los Estados y regiones de la Unión Europea, incluyendo las poblaciones respectivas. Nuestra pretensión es ofrecer una evaluación global del FSE, instrumento concebido para ejecutar programas, proyectos e intervenciones sociales y económicas, que permitan a los grupos de población socialmente vulnerables, en riesgo de exclusión social o en situación de pobreza, salir de del gueto de la marginación. El trabajo se contextualiza en el periodo de la crisis financiera, económica y social surgida en 2008 hasta 2013. Se trata de un segmento temporal coincidente con la vigencia de las Perspectivas Financieras 2007-2014 de la Unión Europea.

Palabras clave: Fondo Social Europeo; Cohesión europea; Evaluaciones; Empleo; Regiones; Inclusión social

\begin{abstract}
Social cohesion policy is defined as a supranational political framework of social solidarity and competitive cooperation which translates into hundreds of thousands projects financed by the European Regional Development Fund (ERDF) and the European Social Fund. A main purpose of these financial instruments is to build physical capital and human capital for European regions and populations affected by the economic and social exclusion, to build and deploy the capabilities and competencies aimed at achieving decent and coherent socio-economic level through their incorporations as active players in the productive system and the labor market. What sets the policy of European social cohesion of other policies, is that legal and financial configuration does not subsidizes poverty, its purpose is to act on the structural causes of social and economic exclusion. Economic and social cohesion described by Member States in the Single European Act of 1986 aims to reduce the structural gaps between states and regions of the European Union, including the respective populations. Our aim is a comprehensive evaluation of the ESF instrument designed to implement programs, projects and social and economic interventions, to enable socially vulnerable groups of population at risk of social exclusion or poverty, out of the ghetto offer marginalization. The work is contextualized in the period of the financial, economic and social crisis that erupted in 2008 until 2013. This is a matching time segment with the duration of the Financial Perspectives 2007-2014 of the European Union.
\end{abstract}

Key Words: European Social Fund; European cohesion; Evaluations; Employment; Regions; Social Inclusion 


\section{Introducción}

Desde mediados de la década de 1990, se ha llevado a cabo un elevado número de investigaciones que utilizan técnicas econométricas (Bradley, 1992) para evaluar los efectos de la política de cohesión sobre el crecimiento de las regiones y el grado de convergencia del Producto Interior Bruto (PIB) per cápita hacia la media de la Unión Europea (Barca, 2009). La mayoría de estos estudios se centran, primordialmente, en periodos de programación anteriores y en el impacto ejercido por la política en las regiones de la Unión Europea formado por 15 países miembros (UE-15). Entre las investigaciones más recientes, son muy pocas las que también abarcan a los países de la UE-12. Estos trabajos emplean diferentes técnicas para generar estimaciones de los efectos de la política, tratando de separar dichos efectos de los producidos por otros factores (Ederveen, Gorter, de Mooij \& Nahuis, 2003).

En torno a la mitad de los estudios realizados han encontrado efectos significativamente positivos de la política de cohesión sobre el crecimiento de la UE (Wostner \& Šlander, 2009), y una cuarta parte, aproximadamente, ha detectado efectos positivos algo menos notables, y no en todos los casos. Por lo tanto, cerca de una cuarta parte de los estudios disponibles han concluido que la política tuvo escasos efectos, o que estos no fueron estadísticamente significativos. Muchos de ellos, no obstante, fueron publicados entre 1996 y 2004, cuando existían mayores limitaciones en cuanto a los datos disponibles y éstos abarcaban un periodo de tiempo más breve.

La mayor parte de los estudios publicados a partir de 2005, que se basan en un conjunto de datos más amplio que abarca un periodo de tiempo más extenso, han llegado a la conclusión de que la política ha tenido importantes resultados positivos. Esta conclusión es común a los estudios que abarcan la UE-12 así como los que engloban a la UE-15. Sin embargo, a pesar de que la mayoría de los estudios llegan a la conclusión de que la política de cohesión ha ayudado a reducir las disparidades regionales en términos de resultados económicos, también señalan que los efectos de dicha política no son uniformes y que son muchos los factores diferentes que influyen en el éxito o el fracaso de la política en un contexto particular, así como en la dimensión del efecto que consigue. Estos factores están relacionados, en particular, con las instituciones existentes, la eficiencia de la gobernanza, las políticas nacionales aplicadas y el desempeño de las regiones vecinas (Comisión Europea, 1999). De igual modo, existen pruebas recientes que apuntan a que los resultados de la política se ven afectados por la forma en que se distribuyen los fondos y se asignan estos entre las diferentes esferas de política, un aspecto fundamental de las reformas recientes.

En el periodo de programación 2000-2006, se gastaron más de 250.000 millones de euros en la UE-15 en el marco de la política de cohesión, y en ayudas a la preadhesión e intervenciones estructurales en la UE-10 (Barca \& McCann, 2011).El gasto ejecutado en los Estados miembros ascendió a 186.000 millones de euros.

El presupuesto total para el periodo de programación 2007-2013 es de 336.000 millones de euros, de los que 173.900 millones han sido asignados a los Estados miembros que se han incorporado a la UE a partir de 2004, 76.000 millones a España, Grecia y Portugal y 26.000 millones a los Lander orientales de Alemania y a la región del Mezzogiorno en Italia como así refiere en el año 2009 la Comisión para la Medición del Desempeño Económico y el Progreso Social (CMEPSP).

Revista de Evaluación de Programas y Políticas Públicas | Núm. 7 (2016), pp. 48-63 
Evaluación del Fondo Social Europeo $2007-2013$ en el contexto

de la política de cohesión europea y la crisis

La crisis ha tenido un impacto profundo en las regiones y ciudades de toda la UE. Las disparidades económicas regionales, que se venían reduciendo, dejaron de hacerlo. El desempleo, por su parte, ha aumentado con rapidez en toda la UE. La pobreza y la exclusión también se han agravado, incluso en muchas ciudades de los Estados miembros más desarrollados. Los Fondos Estructurales y de Inversión Europeos (Fondos ESI) constituyen un medio cada vez más importante para el logro de los objetivos de la Estrategia Europa 2020, sobre todo las metas relacionadas con el empleo y la reducción de la pobreza. En varios Estados miembros, estos fondos se han convertido en la principal fuente de financiación de la inversión pública, ámbito en el que los Estados miembros han aplicado recortes con el fin de reducir sus déficits presupuestarios. Estos cambios, unidos a una mayor orientación a los resultados, se orientan a garantizar que la política de cohesión aborde mejor las disparidades regionales observadas en términos de resultados económicos para ayudar a materializar los objetivos de la Estrategia Europa 2020. Aunque los gobiernos nacionales han tenido que aplicar recortes en el gasto en los últimos años para equilibrar sus presupuestos y que la financiación privada se haya agotado debido a la crisis financiera y económica, la financiación de la política de cohesión ha seguido fluyendo a los Estados miembros y las regiones, apoyando inversiones vitales para el crecimiento y el empleo.

La crisis ha tenido un profundo impacto en los presupuestos nacionales y regionales, limitando la disponibilidad de financiación en todos los ámbitos de inversión. En el conjunto de la UE, la inversión pública se redujo un $20 \%$ en términos reales entre 2008 y 2013. En Grecia, España e Irlanda, la reducción fue de alrededor del 60 \%. En los países de Europa Central y Oriental, donde la financiación de la política de cohesión es particularmente importante, la inversión pública (medida como formación bruta de capital fijo) se redujo en un tercio. Sin la política de cohesión, las inversiones en los Estados miembros más afectados por la crisis habrían registrado un descenso adicional del 50 $\%$. Los fondos de cohesión representan actualmente más del $60 \%$ del presupuesto de inversión en estos países (Comisión Europea, 2014).

La crisis económica ha invertido una prolongada tendencia de convergencia del PIB y la tasa de desempleo dentro de la UE, afectando, en particular, a las regiones del sur de Europa y ha traído consigo un aumento de la pobreza y exclusión social. A su vez, esto ha dificultado la consecución de algunos de los objetivos de la Estrategia Europa 2020. Por ejemplo, en 210 de las 277 regiones de la UE, se registró un aumento del desempleo entre 2007 y 2012. En cincuenta de estas regiones, la tasa de desempleo se ha más que duplicado. La situación es especialmente preocupante para los jóvenes, ya que, en 2012, la tasa de desempleo juvenil fue superior al $20 \%$ en aproximadamente la mitad de las regiones. Como consecuencia de ello, muchas regiones aún no han podido contribuir a la realización del objetivo principal de Europa 2020 de lograr, de aquí a 2020, una tasa de empleo del 75 \% para la población con una edad comprendida entre los 20 y 64 años (Acemoglu, Johnson \& Robinson, 2005).

Además de seguir luchando contra los obstáculos estructurales a largo plazo que afectan al desarrollo, la Comisión y los Estados miembros han respondido a la crisis reorientando algunas inversiones de la política de cohesión a ámbitos en los que los efectos en la actividad económica y el empleo serían directos e inmediatos. En consecuencia, a finales de 2013 se habían reasignado más de 45.000 millones EUR, es decir, el $13 \%$ del total de los fondos. Esta transferencia de fondos ha permitido apoyar medidas destinadas a 
paliar el aumento del desempleo y de la exclusión social y a apoyar la inversión en innovación e investigación y desarrollo (I+D), las ayudas a las empresas, la energía sostenible y las infraestructuras sociales y educativas.

Entre 2007 y 2012, el Fondo Europeo de Desarrollo Regional (FEDER) creó cerca de 600.00o puestos de trabajo. Esto equivale a casi el $20 \%$ del número estimado de empleos perdidos en el mismo periodo, desde el comienzo de la crisis financiera. A su vez, y en el mismo periodo, el Fondo Social Europeo (FSE) aportó 68 millones de participaciones individuales en proyectos. Gracias a las ayudas del FSE, 5,7 millones de parados, o personas inactivas encontraron un empleo y se adquirieron casi 8,6 millones de cualificaciones. Se registraron más de 400.000 empresas emergentes y nuevos empleos por cuenta propia. Medidas que han contribuido a limitar la caída del PIB en muchos países o a evitar un nuevo aumento del desempleo. Los efectos de éstas inversiones se dejarán sentir en los próximos años porque los Estados miembros tienen de plazo hasta finales de 2015 para utilizar los fondos de los Programas del periodo 2007-2013, y porque existe un desfase entre el momento en que se realiza la inversión y el momento en que su impacto es cuantificable (Barca, McCann \& Rodriguez-Pose, 2012).

Con un presupuesto total de más de 450.000 millones de euros (incluida la cofinanciación nacional) para el periodo de programación 2014-2020, la política de cohesión será el principal instrumento de inversión de la UE. Aportará la mayor contribución para apoyar a las PYME, la I+D y la innovación, la educación, la economía con bajas emisiones de carbono, el medio ambiente, la lucha contra el desempleo y exclusión social, el desarrollo de las infraestructuras que conecten a los ciudadanos de la UE y a la modernización de las administraciones públicas. Sus inversiones, combinadas con las reformas estructurales, desempeñaran un papel clave a la hora de apoyar el crecimiento y la creación de puestos de trabajo y en la consecución de los objetivos de la Estrategia Europa 2020 de crecimiento inteligente, sostenible e integrador. El reto es garantizar que estos recursos se utilicen de la manera más eficaz y eficiente posible, maximizando su impacto, consolidando la recuperación y ayudando a la UE a salir de la crisis más fuerte y competitiva que antes.

La política de cohesión está plenamente en consonancia con la Estrategia Europa 2020 y sus objetivos principales en materia de empleo, investigación y desarrollo, cambio climático y energía, educación y lucha contra la pobreza y la exclusión social y, por lo tanto, las inversiones en el marco de la política de cohesión se utilizarán asimismo para apoyar las políticas aplicadas por los Estados miembros de conformidad con las directrices integradas y los programas nacionales de reforma, así como para seguir las Recomendaciones Específicas por País (REP) formuladas por el Consejo. La Comisión también podrá solicitar a los Estados miembros que modifiquen sus acuerdos de asociación y programas operativos para hacer frente a los nuevos desafíos identificados en las REP.

\section{El FSE durante la crisis hasta 2013}

El FSE es el principal instrumento con el que Europa apoya la creación de empleo, ayuda a las personas a conseguir mejores puestos de trabajo y garantiza oportunidades laborales más justas para todos los ciudadanos de la UE. Para ello, el FSE invierte en capital 
Evaluación del Fondo Social Europeo $2007-2013$ en el contexto

de la política de cohesión europea y la crisis

humano europeo: trabajadores, jóvenes y todas aquellas personas que buscan empleo. La financiación del FSE, que asciende a 10.000 millones de euros al año, presupuesto que mejora las perspectivas laborales de millones de europeos, en especial de quiénes tienen dificultades para encontrar trabajo.

La Unión Europea tiene el compromiso de crear más y mejores empleos y de contribuir a una sociedad más inclusiva. Estos objetivos, constituyen el núcleo de la estrategia Europa 2020 que busca generar un crecimiento inteligente, sostenible e inclusivo en la UE. Desafío que resulta aún más exigente en el actual contexto de crisis económica y en el que el FSE desempeña un papel importante a la hora de conseguir los objetivos europeos y mitigar las consecuencias de la crisis económica, sobre todo el aumento de los niveles de desempleo y pobreza.

La Comisión Europea y los Estados miembros de la UE establecen de forma conjunta las prioridades del FSE y deciden el uso que se da a sus recursos. Una de las prioridades es impulsar la capacidad de adaptación de los trabajadores ayudándoles a adquirir nuevas capacidades y la de las empresas fomentando nuevas formas de trabajo. Otras prioridades se centran en mejorar el acceso al empleo, apoyando a jóvenes estudiantes en su transición al mundo laboral, o formando a las personas menos cualificadas que buscan empleo para mejorar sus perspectivas laborales. De hecho, las oportunidades de formación profesional y aprendizaje permanente, dirigidas a dotar a las personas de nuevas cualificaciones, son un componente muy importante de muchos proyectos del FSE. Asimismo, también constituye un objetivo prioritario ayudar a personas de grupos desfavorecidos a encontrar trabajo, fin que contribuye a reforzar la «inclusión social» ya que el empleo cumple un papel crucial en la integración social y en la vida cotidiana. La crisis financiera ha obligado a redoblar los esfuerzos por ayudar a las personas a mantener su empleo o, cuando lo pierden, a reincorporarse al mercado laboral lo más rápidamente posible.

El FSE no es una agencia de empleo y no publica ofertas de trabajo. Lo que sí hace es financiar decenas de miles de proyectos relacionados con el empleo a escala local, regional y nacional en toda Europa: desde pequeños proyectos llevados a cabo por organizaciones de barrio para ayudar a personas con discapacidad a encontrar un trabajo adecuado, hasta proyectos nacionales para promover la formación profesional en el conjunto de la población. Los proyectos del FSE son extraordinariamente variados en cuanto a naturaleza, magnitud y objetivos, y se dirigen a una amplia variedad de grupos. Hay proyectos dirigidos a sistemas educativos, profesorado y niños en edad escolar; a solicitantes de empleo jóvenes y de edad avanzada, y a emprendedores en potencia con todo tipo de antecedentes personales. Las personas son el eje del FSE.

El impacto que tuvo la crisis económica sobre el empleo es muy diferente entre unos países y otros, reflejó el modo en que los distintos sectores se vieron afectados por ella y las medidas políticas diseñadas para hacerle frente. Entre el tercer trimestre de 2008 y el tercer trimestre de 2009 se perdieron más de 5 millones de puestos de trabajo en la UE-27, aunque su distribución fue muy desigual. A partir de 2009 se observa que cada país continuó evolucionando de forma diferente; en algunos de ellos creció la economía, mientras que en otros prosiguió su caída. Teniendo en cuenta que en varios países el empleo disminuyó menos que el PIB durante el periodo de crisis, es posible que todavía no se hayan notado todos los efectos de la recesión económica. El papel del FSE en 
respuesta a la crisis varió en toda la UE según el grado de afectación de los mercados laborales, las ayudas ya existentes y las medidas específicas adoptadas en los diferentes países.

Esta sección ofrece una descripción general de los resultados notificados por los programas ejecutados en el marco de la política de cohesión en sus informes anuales de ejecución. Cada Estado miembro, en colaboración con la Comisión Europea, acuerda uno o varios programas operativos para la financiación del FSE para un periodo de programación de siete años. Estos programas operativos describen las prioridades de las actividades del FSE y sus objetivos.

La UE distribuye la financiación del FSE entre los Estados miembros y las regiones para que financien sus programas operativos. Estos programas apoyan económicamente proyectos relacionados con el empleo que llevan a cabo distintas organizaciones públicas y privadas denominadas beneficiarios. Así se ayuda a los participantes, personas en su mayoría, aunque también pueden ser empresas u organizaciones de otro tipo.

La Comisión Europea y las autoridades nacionales y regionales se asocian para diseñar y gestionar el Fondo Social europeo. Esta asociación también implica la participación de muchos otros colaboradores en el diseño de la estrategia del FSE y en la supervisión de su ejecución como ONGs u organizaciones de trabajadores. Trabajar de manera conjunta es la mejor forma de asegurar que los fondos se invierten de la manera más eficaz y eficiente posible, y que satisfacen las necesidades de la región o comunidad en cuestión. Hay otros dos principios importantes que orientan el funcionamiento del FSE:

- La cofinanciación. Asegura la propiedad a escala nacional y regional: la financiación del FSE siempre debe ir acompañada de financiación pública o privada. Los porcentajes de cofinanciación varían entre el 50 \% y el $85 \%$ (hasta el $95 \%$ en casos excepcionales) del coste total del proyecto, en función de la riqueza relativa de la región.

- La gestión compartida. Permite asumir responsabilidades a los niveles adecuados: las directrices del FSE se diseñan a escala europea tras consultar con un amplio espectro de partes interesadas, y los programas operativos se negocian entre las autoridades nacionales y la Comisión. Las autoridades relevantes de cada país llevan a cabo la ejecución mediante programas operativos.

El nivel de financiación del FSE y los tipos de proyectos que se financian difieren de una región a otra en función de su riqueza relativa, se canaliza a través de los Estados miembros y las regiones. Las regiones de la UE están clasificadas en tres categorías de financiación, basadas en su PIB regional per cápita en comparación con la media de la UE (UE de los 27). Subvenciones que abordan una gran variedad de proyectos que mejoran las perspectivas de empleo de los ciudadanos y los trabajos que éstos desempeñan.

\subsection{Intervenciones constructoras de capital humano y de capacidades institucionales}

En el periodo 2007-2013, las ayudas del Fondo Social Europeo (FSE) equivalieron aproximadamente al $20 \%$ del gasto total en políticas activas de mercado laboral en los Estados miembros, si bien este porcentaje oscilaba entre el $2 \%$ en los países de elevada renta y más del $100 \%$ en las de renta baja (es decir, en las regiones de convergencia). 
Evaluación del Fondo Social Europeo $2007-2013$ en el contexto

de la política de cohesión europea y la crisis

Hasta finales de 2012, el FSE apoyó al menos 19,6 millones de participaciones en programas cuya finalidad era mejorar el acceso de los ciudadanos al empleo; de ellos, unos 3,3 millones de personas encontraron un puesto de trabajo con posterioridad a su participación en dichos programas. En muchos países la crisis provocó que los ciudadanos tuvieran mayores dificultades para encontrar un puesto de trabajo y conservarlo, lo que llevó a modificar algunos de los programas. No obstante, en la mayoría de los Estados miembros los porcentajes de personas que encontraron un empleo y los de aquellas que conservan dicho puesto de trabajo al cabo de 6 o 12 meses se han situado cerca de los objetivos fijados. Además, se informó de 497.00o casos de adquisición de cualificaciones, y un total de 42.00o personas se establecieron como trabajadores autónomos. También se proporcionó ayuda para las personas que ya tenían un empleo, especialmente a las personas con discapacidad y a otros colectivos desfavorecidos.

A su vez, más de 20 millones de jóvenes menores de 25 años habían recibido algún tipo de ayuda, lo que representa un 30 \% del total. Sin embargo, en los Estados miembros del sur de la UE, el porcentaje fue inferior al indicado a pesar del gran número de jóvenes que no se encontraban trabajando, estudiando ni formándose, lo que refleja unas cifras aún mayores de personas con 25 años o más que no tienen trabajo (Accetturo, de Blasio \& Ricci, 2014).

Las evaluaciones realizadas en cinco Estados miembros (Austria, la Republica Checa, Francia, Italia y Portugal) indican que los programas ejecutados en el marco de la política de cohesión prestaron una mayor atención a los jóvenes tras el estallido de la crisis. Todos estos países dieron prioridad a ayudar a los jóvenes en riesgo de abandonar el sistema escolar de forma prematura o que ya lo habían abandonado, y cuatro de ellos (todos excepto Portugal) priorizaron el apoyo a los jóvenes que no se encontraban estudiando, trabajando ni formándose. A partir de 2009 se utilizó una mayor cantidad de recursos para apoyar el autoempleo y la creación de empresas, así como para desarrollar mercados de trabajo intermedios, que proporcionan puestos de trabajo. Pese a que algunos expertos argumentan que los objetivos establecidos no eran particularmente ambiciosos, es necesario ponderar esta afirmación teniendo en cuenta el grave deterioro sufrido por el mercado laboral con respecto al momento en el que se definieron dichos objetivos.

En el periodo 2007-2013 se dio mayor peso al objetivo de inclusión social en comparación con los periodos de programación anteriores. El FSE apoyó diferentes medidas que proporcionaban «itinerarios de inserción» y posibilitaban la reinserción de los colectivos desfavorecidos en el mercado de trabajo. Hasta finales de 2012 se habían invertido 12.900 millones de euros en medidas de inclusión social y se habían comprometido 10.300 millones de euros adicionales. Los resultados de estas medidas solo están disponibles para algunos Estados miembros, aunque los datos disponibles indican que un número sustancial de personas (más de 164.000) encontraron empleo (la mayoría de ellas en España). La cifra de personas que adquirieron una cualificación también es muy importante: de acuerdo con la información disponible, se habrían notificado 148.000 casos de personas que adquirieron cualificaciones.

Las ayudas también se destinaron a combatir la pobreza entre los grupos más vulnerables, como los migrantes, las minorías étnicas y las madres solteras, así como a contribuir a la lucha contra la discriminación. En este contexto, se ayudó a los colectivos afectados a encontrar un trabajo, se realizaron campañas entre la población para eliminar la 
discriminación, se organizaron seminarios sobre la diversidad dirigidos a empresarios y directores de recursos humanos y se impartió formación a los trabajadores de agencias de empleo. En algunos países, más de la mitad de los fondos fueron destinados a ayudar a las mujeres, como en Polonia (56,5 \%), mientras que en otros la proporción fue muy inferior a la mitad (en el Reino Unido, por ejemplo, fue de tan solo un 39,5 \%). En el extremo superior se sitúa España, que informó que, hasta finales de 2011, cerca de 888.000 mujeres habían conseguido un puesto de trabajo tras participar en programas cofinanciados, lo que representa un $62 \%$ de las participantes.

En comparación con el periodo 2000-2006 (Torres e Izquierdo, 2011), en el periodo 2007-2013 se destinó un mayor volumen de fondos (1.000 millones de euros en total) para ayudar a inmigrantes y personas pertenecientes a minorías a encontrar trabajo, así como otros 5.000 millones para otras medidas dirigidas a estos mismos colectivos. Además, se asignaron 10 ooo millones de euros a medidas generales para los colectivos desfavorecidos, incluidos los inmigrantes y las minorías. Hasta finales de 2012, en torno a 6,4 millones de personas pertenecientes a estos dos grupos habían participado en programas financiados por el FSE (Bähr, 2008). Se contabilizaron casi 25,9 millones de participaciones en medidas dirigidas a incrementar el capital humano financiadas por el FSE hasta finales de 2012.

En 13 Estados miembros, el FSE apoyó la modernización de la educación y la formación, para lo que asigno más de 8.000 millones de euros para el diseño, la introducción y la aplicación de reformas. En términos globales, alrededor del $10 \%$ del presupuesto total (35.00o millones de euros) se destinó al capítulo de educación y formación; además, se estima que, hasta finales de 2010, un total de 5 millones de jóvenes, 5,5 millones de personas con bajas cualificaciones y 576.000 personas mayores habían participado en actividades de aprendizaje permanente cofinanciadas9. Aunque estos datos no se pueden agregar debido al problema de la doble contabilidad, ofrecen una indicación de la dimensión de las cifras de participación (Cantwell, 2006). A pesar de que las cifras varían según las características de los participantes y la situación del mercado laboral en cada país, se calcula que, en promedio, entre un $20 \%$ y un $35 \%$ de los beneficiarios encontraron un empleo.

En 2012, la Comisión creó equipos conjuntos de acción juvenil en materia de empleo en los ocho Estados miembros con mayores niveles de desempleo juvenil. Los fondos de la política de cohesión correspondientes al periodo 2007-2013, que todavía no se habían asignado, se utilizaron para incrementar las oportunidades laborales para los jóvenes así como para facilitar el acceso de las PYME a la financiación. Más de 696.00o personas accedieron a un nivel educativo o de formación superior al término de su participación en programas cofinanciados, y se notificaron más de 262.000 casos de personas que habían adquirido cualificaciones, lo que refleja la gran atención prestada a los jóvenes en algunos estados miembros. Además, casi 236.00o participantes obtuvieron un empleo y más de 60.000 se establecieron como trabajadores por cuenta propia. Se espera que más de un millón de jóvenes reciban ayudas gracias a los 4.200 millones de euros asignados (de los que ya se habían comprometido 1.400 millones de euros para diversos proyectos) inmediatamente después de participar en cursos de formación financiados por el FSE.

Para el periodo 2007-2013, las directrices estratégicas comunitarias y el Reglamento del FSE identificaron la buena gobernanza y el desarrollo de las capacidades como cuestiones clave que era necesario abordar, sobre todo en las regiones y los Estados miembros menos desarrollados (Parlamento Europeo, 2006). Como resultado de ello, se destinaron 3.700 millones de euros del FSE al fortalecimiento de la capacidad institucional y de la 
eficiencia de las administraciones y los servicios públicos a escala nacional, regional y local, y, cuando resultaba pertinente, también de los interlocutores sociales y las organizaciones no gubernamentales, con vistas a introducir reformas y a mejorar la regulación y la gobernanza. Estas ayudas se organizaron en torno a dos ejes (Comisión Europea, 2013):

- Mecanismos para mejorar el diseño de las políticas y los programas, el seguimiento y la evaluación a nivel regional y local;

- Desarrollo de las capacidades de ejecución de políticas y programas, incluso en lo referente al cumplimiento de la legislación.

Cuatro Estados miembros (Bulgaria, Rumania, Hungría y Grecia) crearon un programa específico para el fomento de la capacidad, y otros 9 (la Republica Checa, los tres Estados Bálticos, Polonia, Eslovenia, Eslovaquia, Malta, Italia y el Reino Unido, concretamente en Gales) incluyeron este aspecto entre las prioridades de uno de sus programas, sobre todo en programas regionales. Otros países, como Italia, combinaron ambos enfoques, es decir, establecieron un programa nacional específico e introdujeron el aspecto del fomento de la capacidad como eje prioritario en sus programas regionales.

Los programas se centran en cuestiones relacionadas con la estructura de las administraciones, los recursos humanos de éstas y los sistemas y herramientas que emplean. A través de una serie de estudios detallados, se han identificado diversos factores de éxito para un fomento eficaz de la capacidad administrativa:

- Participación de la sociedad civil;

- Metodología y un planteamiento técnico claros;

- Compromiso politico;

- Definición clara de responsabilidades;

- Intercambio de ejemplos de buenas prácticas a escala europea;

- Métodos de seguimiento y evaluación sólidos.

Las cifras anteriores ofrecen una indicación de la dimensión de la actividad respaldada por la política de cohesión y de los tipos de proyectos y medidas cofinanciados. En algunos casos ponen de manifiesto asimismo el resultado del gasto ejecutado y los resultados de las intervenciones. Sin embargo, en sí mismos no muestran los logros de la política de cohesión en términos de valor añadido o la diferencia que ha supuesto dicha política para el desarrollo de las economías regionales o nacionales, el número de personas ocupadas, la calidad de vida de los ciudadanos, etc.

\section{Evaluación de los programas financiados por el FSE}

A continuación, se resumen las conclusiones de las evaluaciones llevadas a cabo a lo largo del periodo 2007- 2013 según las diferentes esferas de política.

Por lo que respecta a la solidez de la medición del impacto de las intervenciones del FSE, un elemento necesario para demostrar la aportación que hacen las intervenciones 
del FSE a sus destinatarios finales, las evaluaciones no fueron capaces, por lo general, de presentar una cantidad significativa de pruebas convincentes. No obstante, de acuerdo con el limitado número de evaluaciones que analizaron algunas intervenciones o programas específicos del FSE, los efectos de las ayudas del Fondo fueron muy importantes y destacables. Estas evaluaciones ponen de manifiesto, por ejemplo, que los participantes en intervenciones apoyadas por el FSE tienen mayor probabilidad de encontrar empleo que las personas integradas en los grupos de control.

En términos globales, los resultados en relación con el aumento de la adaptabilidad, el acceso al empleo y el capital humano se consideraron positivos. Además, algunas evaluaciones sólidas incluían ejemplos de casos en los que las intervenciones habían generado beneficios netos significativos. Por lo que respecta a la inclusión social, los resultados del análisis eran menos concluyentes. El escaso número de pruebas en relación con los resultados y el reducido peso de las pruebas aportadas por las evaluaciones llevó, en general, a la conclusión de que los recursos destinados al capítulo de inclusión social se estaban utilizando de un modo menos coherente y que su eficacia era limitada. La promoción de las asociaciones y el fomento de la capacidad administrativa son ámbitos de política menos comunes en los Estados miembros. Tampoco son muy abundantes las pruebas relativas a los resultados en estas esferas. Pese a todo, las evaluaciones tienden a ser positivas en cuanto a la contribución que han realizado a los servicios públicos.

En las evaluaciones fiables que han llevado a cabo los Estados miembros, éstas ponen de manifiesto que los participantes en intervenciones apoyadas por el FSE tienen mayor probabilidad de encontrar empleo que las personas integradas en los grupos de control. En los Estados miembros en los que las evaluaciones han comparado la actividad de los servicios públicos de empleo con la actividad adicional financiada por el FSE y dirigida al mismo grupo de clientes, se detectaron efectos positivos derivados de los conjuntos de intervenciones respaldadas por el FSE, que en esencia consisten en prestar un servicio más intenso y de mayor calidad a las personas desempleadas. A pesar de ello, los índices de acceso a un puesto de trabajo suelen situarse por debajo del $50 \%$, aunque este porcentaje varía según el tiempo transcurrido al término de una actividad específica. En varios Estados miembros, la proporción de personas que encuentra un trabajo tras participar en alguna iniciativa cofinanciada por el FSE es de un $33 \%$ o menor.

A raíz del estallido de la crisis mundial se generalizo el uso de las subvenciones salariales con el fin de incentivar la contratación de personas desempleadas o pertenecientes a otros colectivos desfavorecidos por parte de las empresas. Sin embargo, las pruebas obtenidas por algunas evaluaciones sugieren que un porcentaje significativo de los beneficiarios finales vuelven al desempleo al vencimiento de dichos contratos. Las evaluaciones realizadas han aportado pruebas de que en las intervenciones del FSE se ha prestado una atención creciente a la igualdad de género en varios países miembros, las intervenciones han contribuido a impulsar la igualdad de género en la agenda política y se han adoptado medidas para las que, de otro modo, no habría existido financiación. Los programas incluyen referencias específicas a medidas para favorecer la conciliación de la vida laboral y la vida privada, el aumento de la participación de las mujeres en el empleo y la reducción de la segregación basada en el género, incluida la brecha salarial. Sin embargo, debido a la incorporación transversal de la perspectiva de género en todos los programas del FSE, resulta difícil estimar el volumen de financiación que se ha destinado al fomento de la igualdad entre hombres y mujeres. No obstante, en términos 
de resultados, las mujeres representaron en torno al $52 \%$ de los beneficiarios de ayudas, si bien esta proporción varía desde el $39 \%$ en el Reino Unido hasta el 56,5 \% en Polonia. Todavía no se dispone de un número significativo de estimaciones de los efectos de estas medidas sobre el empleo, pero en España, por ejemplo, los informes existentes señalan que un total de 888.000 mujeres (casi un $62 \%$ de las participantes) encontraron un empleo hasta finales de 2011 al término de su participación en un programa del FSE. Las medidas de fomento de la igualdad de género respaldadas por el FSE perseguían diversos objetivos (Fondazione G. Brodolini, 2011):

- Aumentar la capacidad de las mujeres para competir en el mercado de trabajo, mediante la mejora de sus cualificaciones;

- Formar a mujeres y hombres en ocupaciones tradicionalmente denominadas por el sexo opuesto, a fin de mejorar sus perspectivas profesionales;

- Ayudar a las mujeres a convertirse en empresarias y proporcionarles servicios de cuidado para facilitarles la conciliación de la vida familiar y laboral;

- Mejorar la calidad de los servicios de cuidado con objeto de alentar a su utilización, y ampliar los horarios de estos servicios; además, formar a personas desempleadas para desempeñar puestos de trabajo en este sector;

- Luchar contra los estereotipos de género y, en menor medida, la segregación de género en el ámbito educativo a través del apoyo a campañas públicas de sensibilización, seminarios dirigidos a las organizaciones sindicales, formación de docentes y padres y revisión de los planes de estudio escolares;

- Apoyar a las mujeres vulnerables y afectadas por la pobreza, que a menudo sufren múltiples tipos de discriminación, así como a las víctimas de la violencia, ayudándolas a adquirir cualificaciones, confianza e independencia económica.

Existen pruebas, en general, de que cada vez es más importante adoptar estrategias multidimensionales que combinen diferentes tipos de intervenciones, con el fin de hacer frente a las múltiples causas de la discriminación o a los diferentes motivos subyacentes a las diferencias entre los géneros. Algunos ejemplos de ello incluyen la combinación de una orientación personalizada con la enseñanza presencial de aptitudes prácticas, la provisión de acceso a ayuda psicológica, la enseñanza de idiomas, la formación profesional y la ayuda en la búsqueda de empleo; es probable que la combinación de estas medidas sea más eficaz que su provisión por separado.

$\mathrm{Al}$ mismo tiempo, el número de medidas destinadas a influir en el contexto social, económico o institucional, o dirigidas al lado de la demanda (como la formación de empresarios o directores de recursos humanos, o la provisión de incentivos a las empresas para contratar mujeres en puestos directivos), fue muy inferior. La evaluación llevada a cabo hizo hincapié en que era necesario intensificar esas medidas con el fin de abordar las causas que se encuentran en el origen de la discriminación.

\subsection{Evaluación de las intervenciones en el ámbito de la inmigración y las minorías}

Una conclusión general de las evaluaciones realizadas por los Estados miembros es que los servicios más eficaces de todos los financiados por el FSE son los que están diseñados 
teniendo en cuenta las necesidades especificase determinados colectivos. En particular, la formación parece ser eficaz en el caso de las personas inmigrantes.

Durante el periodo 2007-2013 se incrementó el volumen de ayudas dirigidas a aumentar la participación en el mercado laboral y la inclusión social de inmigrantes y minorías étnicas con respecto al periodo de programación anterior. Se asignó un presupuesto de 1.170 millones de euros para medidas de ayuda específicas para inmigrantes, y otros 10.000 millones de euros para medidas de carácter general dirigidas a colectivos desfavorecidos, incluidos los inmigrantes y las personas pertenecientes a minorías. Se estima que la mitad de las ayudas beneficiaron a estas últimas. En total, por consiguiente, se destinó un porcentaje ligeramente superior al $8 \%$ del presupuesto global del FSE para ayudar a este colectivo. De acuerdo con los informes presentados, un total de 1,2 millones de personas pertenecientes a este grupo participaron en medidas cofinanciadas por el FSE hasta finales de 2012 (de las que 862.00o eran inmigrantes), aunque la cifra real podría ser unas 100.00o personas mayor debido a que en el caso de las minorías étnicas, especialmente los romaníes, los registros de participación son inferiores a la realidad.

Una evaluación de las ayudas del FSE concluyó que, gracias a dichas ayudas, muchas personas habían encontrado un trabajo mediante el aumento de su empleabilidad, sobre todo por la mejora de su alfabetización, de sus conocimientos en el ámbito de las Tecnologías de Información y Comunicación (TIC) y de sus aptitudes de comunicación; además, otras personas se habían animado a establecerse como trabajadores por cuenta propia. Asimismo, se llegó a la conclusión de que el FSE había ayudado a mejorar los servicios de integración inicial, a crear nuevas redes y estructuras organizativas y, en general, a mejorar la capacidad de los organismos públicos para ayudar a las personas pertenecientes a minorías. Al mismo tiempo, se obtuvieron conocimientos y se intercambiaron experiencias entre los organismos públicos y las ONG con un conocimiento especializado de las necesidades de los inmigrantes y las minorías étnicas y de las barreras a las que se enfrentan cuando tratan de acceder al mercado laboral.

Una evaluación del Programa 2007-2013 del FSE para Inglaterra analizó los efectos de las intervenciones dirigidas a aumentar la empleabilidad de los beneficiarios de la prestación para demandantes de empleo (pagadera durante un periodo máximo de 6 meses) y la prestación por incapacidad o la prestación de empleo y apoyo (pagadera, por lo general, a desempleados de larga duración). La evaluación se basó en datos disponibles a través de la administración pública. El alto número de personas perceptoras de estas prestaciones posibilitó la realización de análisis estadísticos detallados, clasificando los beneficiarios según sus características y el tipo de ayuda recibida. La evaluación concluyó que las medidas de apoyo habían tenido en todo momento efectos positivos en términos de aumento de acceso al empleo y, a su vez, éstos eran más notables en el caso del colectivo más desfavorecido.

Una evaluación de los programas de inserción social dirigidos a personas con discapacidad y ex delincuentes en Lituania estudió los efectos de estos programas desde el punto de vista de la reinserción de los participantes en el mercado laboral. Los datos utilizados permitieron identificar tanto a personas que no habían participado en los programas pese a cumplir los requisitos para ello, así como a otras personas que si participaron en dichos programas. La evaluación concluyó que estos programas incrementaban la probabilidad de que los participantes encontraran un empleo, la duración de este y el salario percibido 
por su desempeño. También descubrió que las intervenciones producían efectos más significativos en las personas con discapacidad que en los ex delincuentes.

Las intervenciones evaluadas se financiaron con cargo al presupuesto correspondiente al periodo de programación 2004-2006. Sin embargo, los datos utilizados en el análisis abarcaron hasta 2010 y el estudio proporcionaba recomendaciones sobre cómo se podría mejorar la utilización de las ayudas estructurales de la UE durante el resto del periodo de programación 2007-2013.

\section{Conclusiones}

Desde que en 1957 se constituyó el Fondo Social Europeo, su evolución y trayectoria hasta el periodo actual constata que para los países miembros de la Unión Europea representa el principal instrumento financiero en el desarrollo de recursos humanos, creación de empleo y acceso al mercado de trabajo. Su distribución de fondos entre regiones, así como la inversión en el capital humano, colabora en la mejora de la calidad de vida de la población europea, especialmente en aquellos grupos que más dificultades encuentran en su inserción en el mundo laboral: personas en exclusión, jóvenes, mujeres,...

Los efectos de la política de cohesión se explican por la colosal inversión que ésta representa en algunos países (hasta el $4 \%$ de su producto interior bruto). En este inicio del período de programación los países se han fijado objetivos concretos y cuantificables, sobre todo en sus respectivos «marcos estratégicos nacionales de referencia». Gestión compartida del FSE con los Estados miembros en el que resulta imprescindible trabajar conjuntamente en una amplia variedad de acciones a nivel nacional, internacional y comunitario estableciendo las prioridades de uso de los recursos orientados a la finalidad de ayudar a recuperar e incrementar el crecimiento económico, garantizar la recuperación del empleo y apoyar el desarrollo sostenible de los Estados.

El desarrollo de una política pública se establece y desarrolla en varias etapas, entre ellas, la evaluación. Fase que determina la consecución de los objetivos, eficacia y eficiencia de un programa pero, además, mide el bienestar y progreso social de la ciudadanía, su impacto en los cambios socio-económicos y ambientales producidos en los países miembros. Los Estados y la Comisión van realizando evaluaciones para medir la repercusión de los programas, analizar su ejecución y comprobar si los recursos ofertados contribuyen a las expectativas planificadas. En la elaboración del informe, los Estados miembros proporcionan los indicadores y datos necesarios para confeccionar las evaluaciones así como la relación de procedimientos comunes y específicos de un programa. Asimismo, se contempla la opción de establecer y organizar un plan de evaluación nacional o multiregional si un país miembro lo considera necesario.

Cada tres años, la Comisión publica su Informe de Cohesión donde describe el desarrollo de las regiones y cómo incide en ellas la política de cohesión. También publica anualmente un informe de situación sobre la cohesión económica y social. Los informes evaluativos, además de evidenciar los resultados y ejecución del programa, además incluye una síntesis de todas las evaluaciones realizadas en el proceso de desarrollo. Sus conclusiones y resultados son considerados en la continuidad del programa o establecer un nuevo plan de acción determinando acciones específicas, personas encargadas de la propuesta y plazos concretos de implementación. 
Para maximizar el impacto de la política de ejecución de las prioridades de la UE, la tendencia es reforzar el proceso de programación estratégica. Esto implica la introducción del Marco Estratégico Común, los contratos de colaboración y un menú de objetivos temáticos de acuerdo con la Estrategia Europa 2020 y sus directrices integradas.

El FSE asume una serie de principios comunes aplicables a todos los fondos estructurales. Estos incluyen la cooperación y la gobernanza multinivel, la conformidad con las leyes nacionales y de la UE aplicables, la promoción de la igualdad entre hombres y mujeres, la no discriminación y el desarrollo sostenible.

La experiencia del Fondo Social Europeo tras la evaluación de la etapa 2007-2013, ha introducido un cambio innovador para el actual periodo de programación 2014-2020, el derecho de suspender todos o parte de los pagos y compromisos determinado por la Comisión Europea cuando un Estado miembro no sea efectivo. Las decisiones sobre suspensiones deben ser proporcionadas y efectivas, y deben tener en cuenta el impacto de los programas afectados dentro de la situación económica y social del Estado miembro relevante.

La evolución del FSE se ha caracterizado por una línea constante de innovación y adaptación a los problemas y necesidades emergentes por lo que, como conclusión final, se puede afirmar que los efectos del Fondo Social Europeo desde su nacimiento hasta el periodo actual arroja resultados positivos, contribuye ampliamente en el desarrollo de políticas sociales y de empleo en la población europea.

\section{Bibliografía}

Accetturo, A., de Blasio, G. \& Ricci, L. (2014). A tale of an unwanted outcome: Transfers and local endowments of trust and cooperation. Journal of Economic Behavior \& Organization. 102 (3), 74-89.

Acemoglu, D., Johnson, S. \& Robinson, J. (2005). Institutions as the Fundamental Cause of Long-Run Growth. In P. Aghion and S. Durlauf (eds), Handbook of Economic Growth, (pp. 385-472). Amsterdam: Elsevier.

Bähr, C. (2008). How does sub-national autonomy aff ect the effectiveness of structural funds? Kyklos, 61(3), 3-18.

Barca F. (2009). An agenda for a reformed Cohesion Policy. A place-based approach to meeting European Union challenges and expectations. Independent report prepared at the request of Danuta Hübner, Commissioner for Regional Policy. Brussels: Commission of the European Communities.

Barca F. McCann P. \& Rodriguez-Pose A. (2012). The Case for Regional Development Intervention: Place-Based versus Place-Neutral Approaches. Journal of Regional Science, 52(1), 134-152.

Barca, F. \& McCann, P. (2011). Outcome Indicators and Targets: Towards a System of Monitoring and Evaluation in EU Cohesion Policy. Recuperado el 28 de marzo de 2016, de http://ec.europa.eu/regional_policy/sources/docoffic/2014/working/ wd_2014_en.pdf 
Bradley, J. (1992). Economic and environmental impact of the Community support framework: Modeling growth and development in the EC periphery, Contract JOU2-CT92-D257. Brussels: Commission of the European Communities.

Cantwell, J. (2006). Innovation and Competitiveness. In Fagerber, J., Mowery, D. And Nelson, R.(eds), The Oxford Handbook of Innovation (pp. 543-568). Oxford: Oxford University Press.

CMEPSP (2009). Report of the commission on the measurement of economic performance and social progress. Recuperado el 28 de marzo de 2016, de http:// www.insee.fr/fr/publications-et-services/dossiers_web/stiglitz/doc-commission/ RAPPORT_anglais.pdf

Comisión Europea (2014). Sexto informe sobre cohesión económica, social y territorial: inversión para el empleo y el crecimiento. Recuperado el 28 de marzo de 2016, de http://eur-lex.europa.eu/legal-content/ES/TXT/ $\mathrm{PDF} /$ ?uri=COM:2014:473:FIN\&rid=5

Comisión Europea (2013).Cohesion Policy: Strategic Report 2013. Recuperado el 28 de marzo de 2016, de http://ec.europa.eu/regional_policy/how/policy/doc/ strategic_report/2013/strat_report_2013_en.pdf

Comisión Europea (1999). The socio-economic impact of projects financed by the Cohesion Fund: A modeling approach. Luxembourg: Office for official publications of the European Communities. Recuperado el 28 de marzo de 2016.

Ederveen S., Gorter J., de Mooij R. \& Nahuis R. (2003). Funds and Games: The Economics of European Cohesion Policy. European Network of Economic Policy Research Institute, paper no. 3. Recuperado el 28 de marzo de 2016, de http:// people.ds.cam.ac.uk/mb65/library/ederveen.a.o.2003.pdf

Fondazione, G. Brodolini (2011). Evaluation of the European Social Fund's support to Gender Equality. Recuperado el 28 de marzo de 2016.

Parlamento Europeo (2006). Reglamento (No 1081/20o6 (Articulo 3.2, letra b). Recuperado el 28 de marzo de 2016, de http://www.empleo.gob.es/UAFSE/es/ normativas/pdf/Reg_1081.pdf

Torres, R. e Izquierdo, J. (2011). Cohesión Euroamericana. Supranacionalidad de los Derechos Sociales. Justicia laboral: revista de Derecho del Trabajo y de la Seguridad Social, 48, 29-46.

Wostner P. \& Šlander S. (2009). The Effectiveness of EU Cohesion Policy Revisited: Are EU Funds Really Additional. Glasgow: University of Strathclyde. Recuperado el 28 de marzo de 2016, de http://www.eprc.strath.ac.uk/eprc/documents/PDF_files/ EPRP_69_The_Effectiveness_of_EU_Cohesion_Policy_Revisited.pdf 